\title{
Home Sleep Monitor for Detecting Apnea Episodes by Nasal Flow and Tracheal Sound Recordings
}

\author{
Wataru Hida, Hiroshi Miki, Yoshibiro Kikuchi, \\ Chimaru Miura, Noriyuki Iwase, Yoshio Shimizu and \\ Tamotsu Takishima \\ The First Department of Internal Medicine, Tohoku \\ University School of Medicine, Sendai 980
}

\begin{abstract}
Hida, W., Miki, H., Kikuchi, Y., Miura, C., Iwase, N., Shimizu, Y. and Takishima, T. Home Sleep Monitor for Detecting Apnea Episodes by Nasal Flow and Tracheal Sound Recordings. Tohoku J. Exp. Med., 1988, 156, Suppl., 137-142 We have developed a portable home sleep monitoring system using nasal airflow (NA), tracheal sound recordings (TSR), and electrocardiogram (ECG). NA was recorded by two thermisters. TSR was recorded by a microphone attached to the skin overlying the cervical trachea. Three kinds of signals were recorded with a cassette recorder. Thirty-seven outpatients who had sleep complaints were monitored duing sleep at home using this recorder. Attachment of the pickups was performed by the patients themselves. Recordings were played back and analyzed by a personal computer to evaluate apnea episodes from TSR and R-R intervals beat by beat. This home monitoring system had labor-saving and cost-saving benefits and seemed to be a satisfactory technique for screening. sleep apnea syndrome; portable sleep monitor; sleep disorder
\end{abstract}

Polysomnography has been used to provide pathophysiologic diagnosis of patients with sleep disorders (Guilleminault et al. 1976, 1980). However, this technique is burdensome, laborious, time consuming, costly and inconvenient for epidemiologic study, because the patients must generally be admitted to the hospital to receive the examination. The technique has, therefore, been unsuitable for screeing outpatients with sleep complaints. Previously, home sleep recording using the inductive plethysmograph has been reported (Ancoli-Israel et al. 1981; Gyulay et al. 1987). However, it seems to be costly and still difficult for the patient himself to use this device. We have developed a portable home sleep monitoring system using nasal airflow, tracheal sound recordings, and electrocardiograms. The focus of this recording method is the detection of sleep apnea in the patient's home.

Correspondence to: Tamotsu Takishima, M.D. Professor and Chairman, the First Department of Internal. Medicine, Tohoku University School of Medicine, 1-1 Seiryo-machi, Aoba-ku 980, Sendai, Japan. 


\section{MetHods}

A block diagram of the home monitoring system is shown in Fig. 1. The upper panel in Fig. 1 shows the four-channel analog recorder, although a fourth channel for clock time is not shown. Tracheal sound was recorded in the first channel by a modification of Cumminskey's method (Krumpe and Cummiskey 1980 ; Cummiskey et al. 1982). Tracheal sounds were measured with a quarterinch microphone (WM-62A, National, Tokyo) attached to the skin overlying the cervical trachea. Sound signals were bandpass filtered (200-1000 $\mathrm{Hz}$ ) to exclude cardiac and vascular sounds, rectified, and integrated to obtain a sound envelope. The second channel recorded nasal airflow (NA) by two thermisters (503ET2, Ishizuka Elect. Corp., Tokyo) placed near the nasal orifices. The third channel recorded electrocardiogram (ECG). This apparatus weighs approximately $800 \mathrm{~g}$, and is operated by a $3-\mathrm{V}$ battery for the amplifier and a $9-\mathrm{V}$ battery for recording. The former battery is rechargeable and the latter is non-rechargeable. This house sleep monitor is light and portable and is capable of recording for $12 \mathrm{hr}$. We explained operation of the monitor to the subjects and, before retiring, they themselves attached the three pickups and started the machine in operation. On the following day, the casette tapes and machine were brought back to the hospital and rocordings were played back on the four channel recorder and analyzed by the personal computer (Model N10, Data General, Tokyo) to display trends in apnea episodes, a histogram of apnea or trends in $R-R$ intarvals. The lower panel in Fig. 1 shows the computer analysis system. Apnea was defined when an intensity less than $15-20 \%$ of mean tracheal sound recording obtained with a two minute moving average filter lasted more than 10 sec. Furthermore, in ECG, pulse waves synchronized to QRS in the second lead were integrated. Thus, $R-R$ interval was converted to a voltage amplitude signal, which was input to the computer. All data were filed in disk.
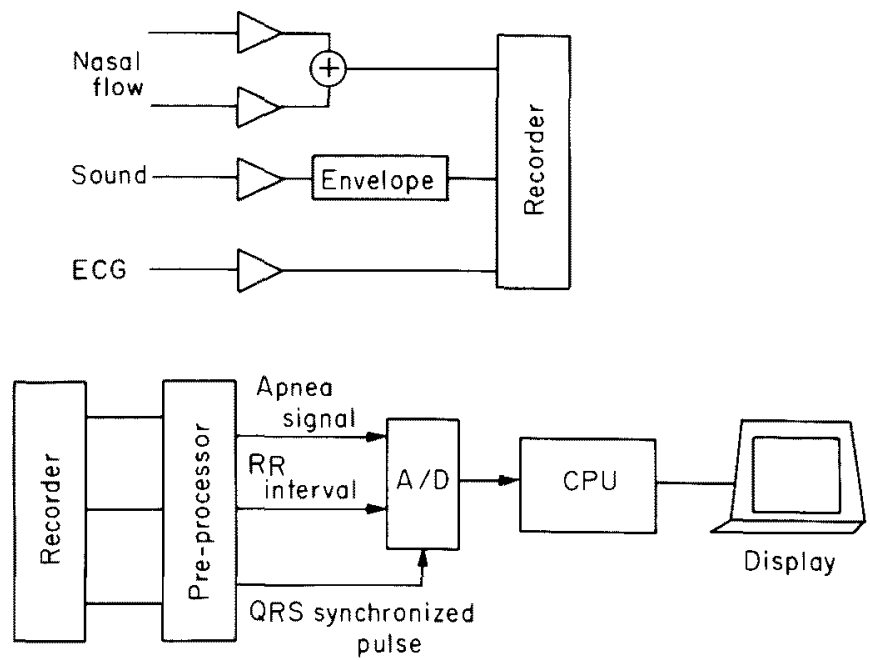

Fig. 1. Block diagram of home sleep monitoring system. Tracheal sound, nasal airflow and electrocardiogram (ECG) were monitored (upper panel), and analyzed by the computer (lower panel). 


\section{RESULTS}

Fig. 2 shows normal tracing of the envelope of tracheal sound, nasal airflow and ECG during sleep in a normal subject. An upward direction of nasal flow indicates the inspiratory direction. Breathing was almost regular and apnea episodes were not found. In this case the total number of apnea episodes on one night was zero. Apnea episodes in one night in other normal subjects were within 10 episodes/night.

Fig. 3 shows tracings of abnormal breathing with repeated apnea episodes. These episodes are characterized by a cessation of nasal airflow and an absence of tracheal sounds.

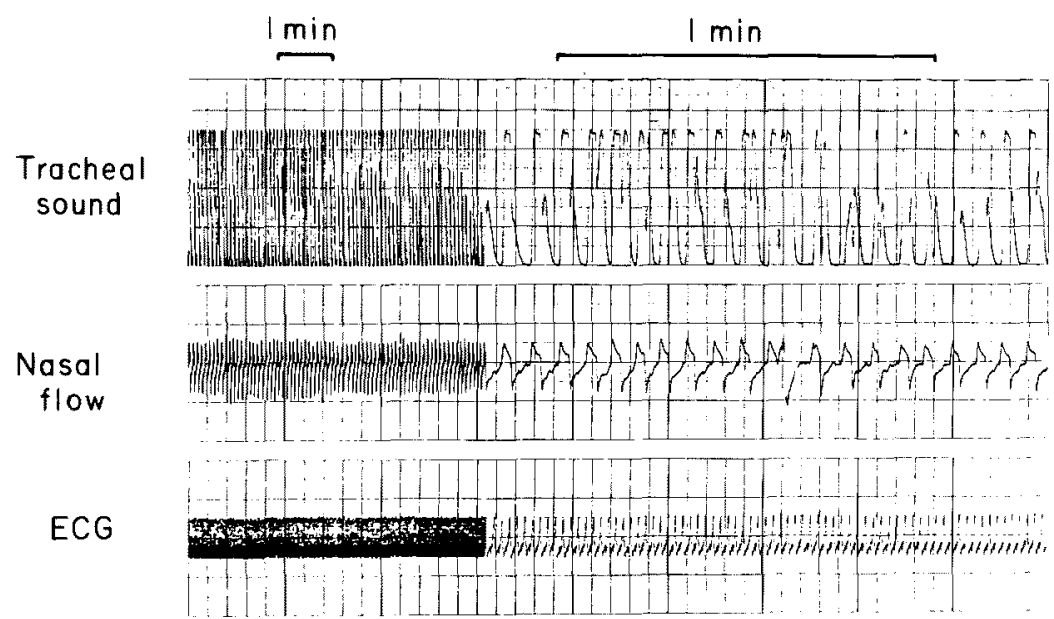

Fig. 2. Tracings of the envelope of normal tracheal sound, nasal airflow and ECG. See text for further explanation.

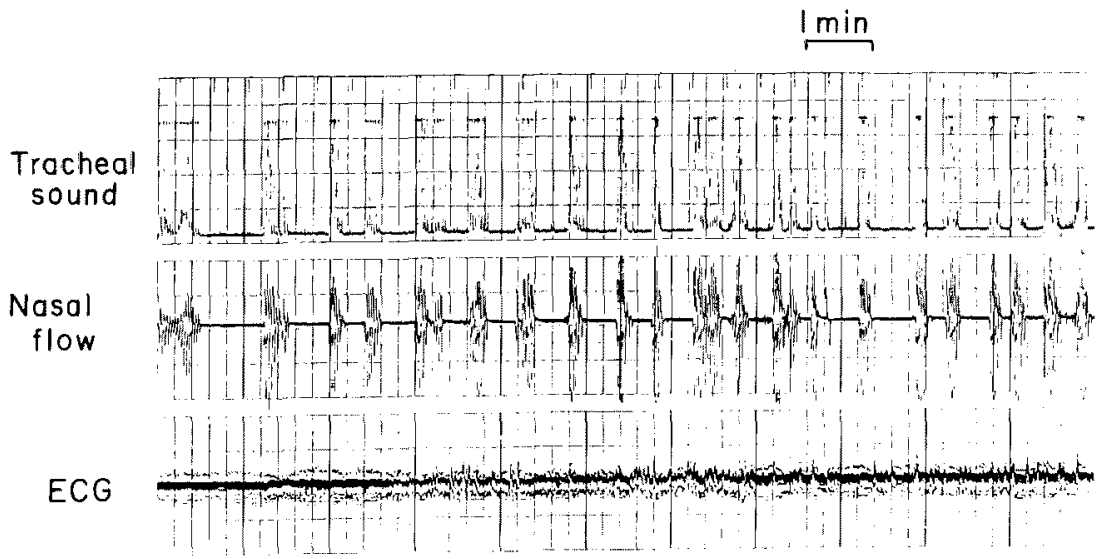

Fig. 3. Tracings showing repeated apnea episodes. See text for further explanation. 


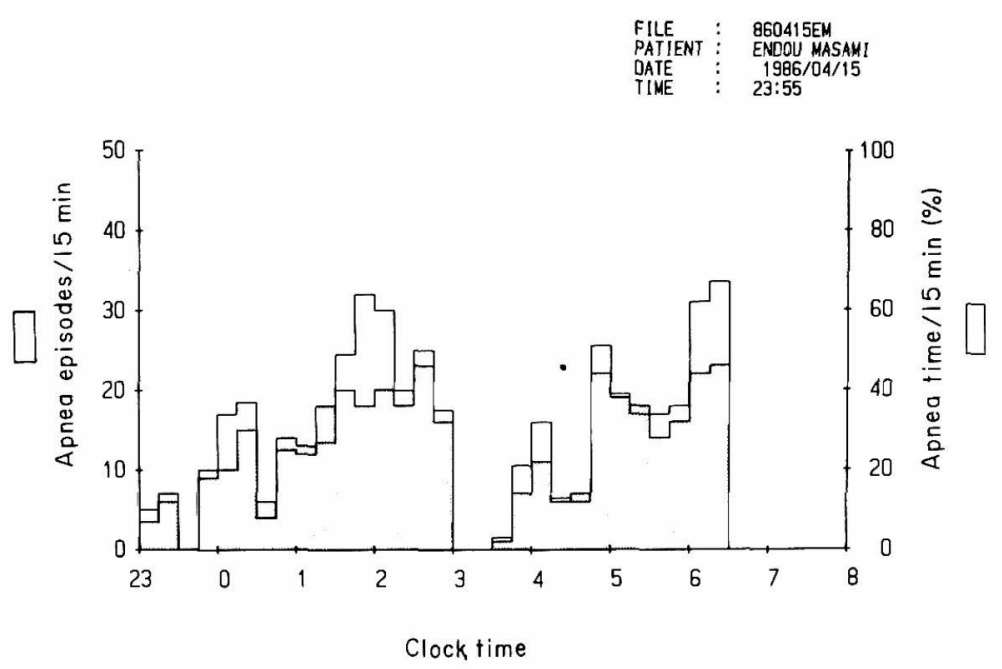

Fig. 4. Display of trend in frequency of apnea episodes per 15 min period (open area) and percent of apnea time per 15 min period (shaded area).

Fig. 4 shows a display of frequency of apnea episodes per $15 \mathrm{~min}$ period and percent of apnea time in consecutive 15 min periods. Both are shown as a function of clock time. In this case, apnea episodes and percent of apnea time exhibited two peaks; one is between 1:00 am and 3:00 am, and another is between $5: 00 \mathrm{am}$ and $6: 30 \mathrm{am}$.

Fig. 5 shows a typical histogram of apnea duration. In this case, main apnea durations were distributed from 10 to 30 sec. Apnea durations over 60 sec were also obtained. The pattern of distribution of apnea episodes was similar to a

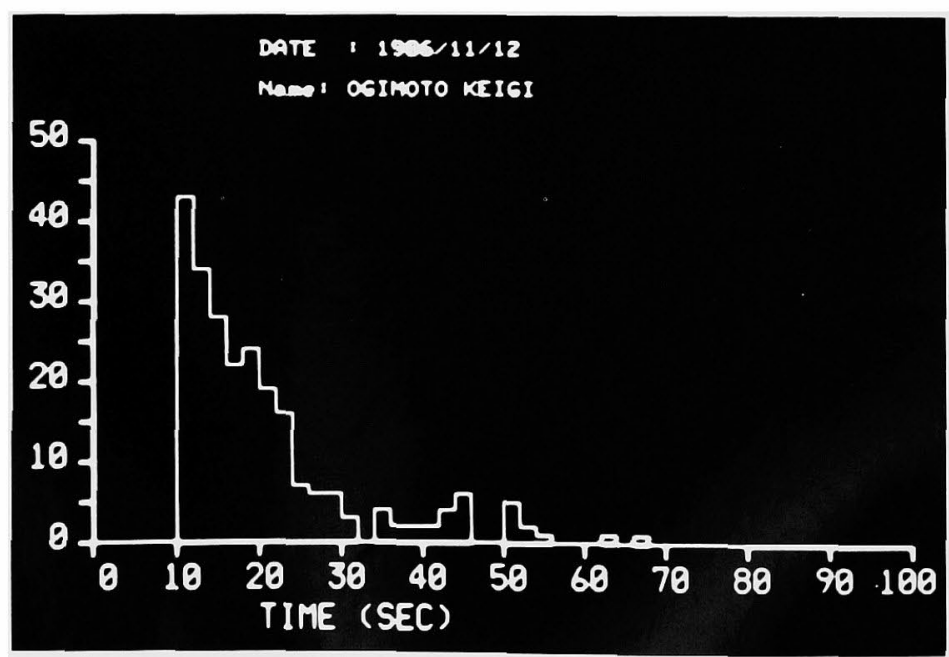

Fig. 5. Photographic display of the apnea duration histogram from the computer.

Vertical axis, number of apnea; horizontal axis, apnea duration. 
Poisson's distribution. In normal subjects, the $R-R$ interval was almost constant, but in patients with apnea episodes, the R-R interval altered periodicaly from tachycardia during breathing to bradycardia during apnea, as previously reported (Motta et al. 1978).

\section{Discussion}

We have developed a portable home sleep monitor using airflow, tracheal sounds and ECG, and analyzed recorded data by a computer to obtain a display of trend in apnea episodes, a histogram of apnea durations and trend in R-R interval.

When apnea episodes estimated by tracheal sound recordings were compared with those estimated by nasal airflow, the former tended to be smaller than the latter, but not significantly different. In the present study, the former was adopted for analyzing sleep apnea. Even when nasal airflow was zero, tracheal sound could be detected when mouth airflow was present.

Although our home sleep monitor did not provide the exact times of the sleep and awake state because of a lack of electroencephalogram (EEG), electrooculogram $(\mathrm{EOG})$ or electromyogram $(\mathrm{EMG})$, we assessed the sleeping or waking time from the patient's judgement. Therefore, apnea indexes from the home sleep monitor may not be correct. Usually the sleeping time reported by patients seemed to be longer than that estimated by EEG. If so, apnea index from the sleep monitor may be underestimated. Nevertheless, total apnea episodes were estimated well from the portable home sleep monitor,

This portable recording technique has the advantage of greater comfort than the home monitor system reported previously (Ancoli-Israel et al. 1981 ; Gyulay et al. 1987), which was not always convenient for attachment of electrodes by patients themselves. Furthermore, since in these previous reports, inductive plethysmography was used to detect apnea episodes, it appeared difficult to calibrate change in volume.

We adopted tracheal sound recording (TSR) for the detection of apnea episodes. From TSR, we could not clarify whether the apnea type is central, obstructive or mixed apnea. Furthermore we could not clarify the breathing pattern. However, it was easy to confirm apnea episodes.

Three kinds of pickups were attached by the patients themselves just before sleeping. With all subjects, attachment of pickups were successful and recordings played back from casette tape were also successful. Furthermore, software for analysis of sleep apnea was appropriate for personal computers which are commonly used in hospitals. Therefore, anyone could analyze sleep data with appropriate software, providing a significant cost-saving. Our portable home sleep monitor seemed to be satisfactory for sleep apnea screeing and clinical follow up, and to be convenient for epidemiologic study. 


\section{References}

1) Ancoli-Israel, S., Kripke, D., Mason, W. \& Messin, S. (1981) Comparisons of home sleep recordings and polysomnograms in older adults with sleep disorders. Sleep, 4, $483-491$.

2) Cummiskey, J., Williams, T.C., Krumpe, P.E. \& Guilleminault, C. (1982) The detection and quantification of sleep apnea by tracheal sound recordings. Am. Rev. Respir. Dis., 126, 221-224.

3) Guilleminault, C., Tilkian, A. \& Dement, W.C. (1976) The sleep apnea syndrome. Ann. Rev. Med., 27, 465-484.

4) Guilleminault, C., Cummiskey, J. \& Dement, W.C. (1980) Sleep apnea syndorome. In: Advances in Internal Medicine, edited by C.H. Stollerman, Vol. 26, Year Book Medical Publications, Inc., Chicago, pp. 347-374.

5) Gyulay, S., Gould, D., Sawyer, B., Pond, D., Mant, A. \& Saunders, N. (1987) Evaluation of a microprocessor-based portable home monitoring system to measure breathing during sleep. Sleep, 10, 130-142.

6) Krumpe, P.E. \& Cummiskey, J. (1980) Use of laryngeal sound recordings to monitor apnea. Am. Rev. Respir. Dis., 122, 797-801.

7) Motta, J., Guilleminault, C., Schroeder, J.S. \& Dement, W.C. (1978) Tracheostomy and hemodynamic changes in sleep-induced apnea. Ann. Intern. Med., 89, 454-458. 